\title{
Solving Adaptive Image Restoration Problems via a Modified Projection Algorithm
}

\author{
Hao Yang, ${ }^{1,2}$ Xueping Luo, ${ }^{3}$ and Leiting Chen $^{1,2}$ \\ ${ }^{1}$ School of Computer Science and Engineering, University of Electronic Science and Technology of China, \\ Chengdu, Sichuan 611731, China \\ ${ }^{2}$ Sichuan Provincial Key Laboratory of Digital Media, Chengdu, Sichuan 611731, China \\ ${ }^{3}$ College of Computer Science and Technology, Southwest University for Nationalities, Chengdu, Sichuan 610041, China
}

Correspondence should be addressed to Hao Yang; vhaoyang@gmail.com

Received 26 January 2016; Accepted 9 March 2016

Academic Editor: Daniel Zaldivar

Copyright (C) 2016 Hao Yang et al. This is an open access article distributed under the Creative Commons Attribution License, which permits unrestricted use, distribution, and reproduction in any medium, provided the original work is properly cited.

We introduce a new general TV regularizer, namely, generalized TV regularization, to study image denoising and nonblind image deblurring problems. In order to discuss the generalized TV image restoration with solution-driven adaptivity, we consider the existence and uniqueness of the solution for mixed quasi-variational inequality. Moreover, the convergence of a modified projection algorithm for solving mixed quasi-variational inequalities is also shown. The corresponding experimental results support our theoretical findings.

\section{Introduction}

Digital image restoration plays an important role in many applications of sciences and engineering such as medical and astronomical imaging, film restoration, and image and video coding. Recovering an image from a degraded image is usually an ill-posed inverse problem and it should be dealt with through selecting a suitable regularizer. Since the work of Rudin, Osher, and Fatemi (ROF) in [1], the regularization methods based on total variation (TV) have known a success, mostly due to their ability to preserve edges in the image. In recent years, a number of research works have been proposed in the field of TV regularization approaches, which are used for the task of image denoising and nonblind image deblurring. Aujol et al. [2] replaced $L_{2}$ norm of the data fidelity term by $L_{1}$ norm to modify the ROF functional model. The TV regularization approaches in $[3,4]$ can be described by means of locally dependent constraint sets; that is, the functional is adaptive to the input data. Another class of approaches are the nonlocal methods $[5,6]$ including nonlocal variants of TV regularization. [7-9] extended TV regularization to second- or higher-order cases. These works mentioned above considered TV regularization approaches for solving the image denoising problems. In addition, these approaches can also be utilized for the image deblurring; see, for example, [10-12]. Chambolle [10] proposed algorithm for minimizing the TV model and applied the algorithm to image zooming. A TV deblurring approach with adaptive choice of the regularization parameter was presented in [12]. In all these literatures, the image restoration problem is always regarded as optimization problem using discrete TV regularization. How to solve such optimization problem with a TV regularization, which is fundamental and crucial, is the core problem in our discussion.

It is well known that the theory of variational inequality has been developed as a class of important tools for the study of minimization problems; see, for example, [13]. Among such variational inequalities, inverse variational inequalities, mixed variational inequalities, and quasi-variational inequalities are very significant generalizations, which have been applied to a wide range of problems, such as mechanics, economics, finance, optimal control, and transportation. References $[14,15]$ proposed Tikhonov regularization method and a general regularization method for solving inverse 
variational inequality problems. Luo and Yang $[16,17]$ further extended the results of $[14,15]$ to the inverse mixed variational inequality problems. The generalized quasi-variational inequality problem was introduced in [18]. However, to our knowledge, a few works implemented quasi-variational inequality to deal with image restoration problem. Recently, Lenzen et al. [19-21] firstly considered a class of quasivariational inequalities for studying adaptive image restoration, where adaptivity is solution-driven adaptivity. Moreover, they showed that a lot of experimental results support their theoretical findings.

Inspired and motivated by the works of $[19,20]$, in this paper, we introduce a general TV regularization which includes TV regularization of the classical ROF model [1] as its special case. For solving the minimization problem with generalized TV regularization, we discuss its dual problem, which is like the following formulation:

$$
\min _{p \in \mathscr{D}}\{F(p)+\Phi(p)\}
$$

where $\mathscr{D}$ is a convex constraint set. For generalizing the regularization approach to solution-driven adaptivity, we find a fixed point of the following mapping:

$$
p_{0} \longmapsto \bar{p}:=\underset{p \in \mathscr{D}\left(p_{0}\right)}{\operatorname{argmin}}\{F(p)+\Phi(p)\}
$$

The above fixed point problem is equivalent to solving a mixed quasi-variational inequality [22]. We provide the existence and uniqueness of a fixed point for the mixed quasi-variational inequality for adaptive image restoration. Thus, our theoretical results generalize the research works of [19]. Meanwhile, we propose a modified projection algorithm for solving mixed quasi-variational inequality and prove its convergence. Finally, we give improved experimental results compared to the experiments presented in [19]. Moreover, our experimental results show that the solution-driven adaptive generalized TV model produces excellent restoration effects for different test images.

The rest of this paper is organized as follows. In Section 2, we recall some notations concerned with generalized $\Phi$ projection operator. In Section 3, we introduce the generalized TV regularization which covers other TV regularizers given in literature. Our model of solution-driven adaptivity described by means of mixed quasi-variational inequalities is shown in Section 4. We consider the theoretical results in Section 5, where we prove the existence and uniqueness of the solution for mixed quasi-variational inequality. In Section 6, we present a modified projection algorithm and its convergence. We give a lot of numerical experiments supporting our theoretical results and showing our better improvement in Section 7. Finally, we conclude this paper in Section 8.

\section{Preliminaries}

In this section, we recall the concept of the generalized $\Phi$ projection operator, together with its properties.
Let $\Omega$ be a nonempty closed convex subset of $\mathbb{R}^{n}$. Let $G$ : $\mathbb{R}^{n} \times \Omega \rightarrow \mathbb{R} \cup\{+\infty\}$ be a function defined as follows:

$$
G(x, \xi)=\|x\|^{2}-2\langle x, \xi\rangle+\|\xi\|^{2}+2 \rho \Phi(\xi),
$$

where $\xi \in \Omega, x \in \mathbb{R}^{n}, \rho$ is a positive number, and $\Phi: \Omega \rightarrow$ $\mathbb{R} \cup\{+\infty\}$ is a proper, convex, and lower semicontinuous function.

Definition 1. The generalized $\Phi$-projection operator $\Pi_{\Omega}^{\Phi}$ : $\mathbb{R}^{n} \rightrightarrows \Omega$ is defined as

$$
\begin{aligned}
& \Pi_{\Omega}^{\Phi}(x)=\left\{u \in \Omega: G(x, u)=\inf _{\xi \in \Omega} G(x, \xi)\right\}, \\
& \forall x \in \mathbb{R}^{n} \text {. }
\end{aligned}
$$

From Lemmas 3.1 and 3.2 of [25], we know that $\Pi_{\Omega}^{\Phi}$ is a single valued and nonexpansive mapping and $x^{*}=\Pi_{\Omega}^{\Phi}(x)$ if and only if

$$
\left\langle x^{*}-x, y-x^{*}\right\rangle+\rho \Phi(y)-\rho \Phi\left(x^{*}\right) \geq 0, \quad \forall y \in \Omega .
$$

\section{Generalized TV Regularization}

In this section, we introduce a new variational approach for image denoising and nonblind image deblurring that is based on total variation regularization. Our general approach covers various adaptive and anisotropic types of TV regularization approaches.

The image deblurring problem formulation is as follows. Let $f \in \mathbb{R}^{n}$ be a degraded noisy image, which is obtained from a noise-free image $u \in \mathbb{R}^{n}$ by convolution with a blurring kernel $M$, followed by an addition of Gaussian noise; that is,

$$
f=M u+\delta
$$

where $M$ is $n \times n$ invertible matrix and $\delta$ is a Gaussian random variable with zero mean. The above problem is a typical inverse problem. In order to recover $u$ from $f$, assuming that $u \mapsto M * u$ is a mapping from $\mathbb{R}^{n} \rightarrow \mathbb{R}^{n}$, we aim at considering the following optimization problem:

$$
\underset{u \in \mathbb{R}^{n}}{\operatorname{argmin}} E(u)=\frac{1}{2}\|M u-f\|_{L^{2}}^{2}+\alpha \operatorname{TV}(u), \quad \alpha>0 .
$$

In particular, if $M:=\mathrm{Id}$, the minimization problem (7) reduces to image denoising problem, where Id denotes an identity mapping.

Now we denote by $L: \mathbb{R}^{\text {nd }} \rightarrow \mathbb{R}^{n}$ the discretization of the divergence operator div and denote by $\|\cdot\|_{2} L_{2}$ norm. Let us define the following generalized total variation regularizer:

$$
\alpha \operatorname{TV}(u):=\sup _{p \in \mathscr{D}}\left\{\langle L p, u\rangle_{L_{2}}-\left\|M^{-\top} L p\right\|_{2}\right\}
$$

where

$$
\mathscr{D}=\left\{p \in \mathbb{R}^{n d}, p_{i} \in B_{\alpha}(0), i=1, \ldots, n\right\}
$$


for $p=\left(p_{1}, p_{2}, \ldots, p_{n}\right)^{\top}$ with $p_{i} \in \mathbb{R}^{d}$ and $B_{\alpha}(0)$ is $d$ dimensional closed ball with radius $\alpha$ centered at 0 . Then the optimization problem is given as

$$
\begin{aligned}
\underset{u \in \mathbb{R}^{n}}{\operatorname{argmin}} E(u)= & \frac{1}{2}\|M u-f\|_{2}^{2} \\
& +\sup _{p \in \mathscr{D}}\left\{\langle L p, u\rangle_{L_{2}}-\left\|M^{-\top} L p\right\|_{2}\right\} .
\end{aligned}
$$

Problem (10) includes a large variety of problems as its special cases:

(i) If $\left\|M^{-\top} L p\right\|_{2}=0$, then (10) reduces to problem (2.12) of [19].

(ii) If $\left\|M^{-\top} L p\right\|_{2}=0$ and $M:=\mathrm{Id}$, then (10) reduces to the classical ROF model of [1].

We derive the corresponding dual problem of (10) as follows. The optimality condition for $u$ reads

$$
M^{\top}(M u-f)+L p=0 .
$$

It follows from (11) that

$$
\begin{aligned}
u & =M^{-1}\left(f-M^{-\top} L p\right), \\
M u & =f-M^{-\top} L p,
\end{aligned}
$$

where $M^{-\top}:=\left(M^{\top}\right)^{-1}$. Using the abbreviation $A:=M^{-\top} L$, from (11) and (12) we obtain the dual problem

$$
\begin{aligned}
E^{*}(p)= & \frac{1}{2}\|M u-f\|_{2}^{2}+\langle L p, u\rangle_{L_{2}}-\left\|M^{-\top} L p\right\|_{2} \\
= & \frac{1}{2}\left\|M^{-\top} L p\right\|_{2}^{2}+f^{\top} M^{-\top} L p \\
& -\left(M^{-1} M^{-\top} L p\right)^{\top} L p-\left\|M^{-\top} L p\right\|_{2} \\
= & \frac{1}{2}\|A p\|_{2}^{2}+f A p-(A p)^{\top} A p-\|A p\|_{2} \\
= & -\frac{1}{2}\left(\|A p\|_{2}^{2}-2 f A p\right)-\|A p\|_{2} \\
= & -\frac{1}{2}\left(\langle A p-f, A p-f\rangle_{L_{2}}-\|f\|_{2}^{2}\right)-\|A p\|_{2} \\
= & -\frac{1}{2}\|A p-f\|_{2}^{2}+\frac{1}{2}\|f\|_{2}^{2}-\|A p\|_{2} .
\end{aligned}
$$

When maximizing $E^{*}(p)$ over $\mathscr{D}$, the constant term $(1 / 2)\|f\|_{2}^{2}$ can be omitted without changing the optimum. Moreover, the maximization of $E^{*}$ equals the minimization of $G(p):=-E^{*}(p)$, and we can formulate the dual problem of $(10)$ as

$$
\begin{aligned}
\underset{p \in \mathscr{D}}{\operatorname{argmin}} G(p) & =F(p)+\Phi(p) \\
& =\frac{1}{2}\|A p-f\|_{2}^{2}+\|A p\|_{2}
\end{aligned}
$$

with $\mathscr{D}:=\mathscr{D}_{\text {loc }}^{1} \times \mathscr{D}_{\text {loc }}^{2} \times \cdots \times \mathscr{D}_{\text {loc }}^{n}$, where each local constraint set $\mathscr{D}_{\text {loc }}^{i}$ is a $d$-dimensional closed ball.

From a solution $\bar{p}$ of the dual problem, we can retrieve the solution $\bar{u}$ of the primal problem by $\bar{u}=M^{-1}(f-A \bar{p})$. Therefore, the key issue in our discussion of generalized TV image restoration problem is to solve the above minimization problem (15).

\section{Solution-Driven Adaptivity}

In [20], Lenzen et al. proposed a kind of adaptivity, where the constraint set $\mathscr{D}$ depends on the unknown solution $p$ of the problem. Naturally, the adaptivity is determined by the noisefree image $u$, which can be obtained by $u=M^{-1}(f-A p)$. Moreover, the experimental results of [19] showed that the adaptivity was improved by solution-driven model. In the following, in order to study generalized TV image restoration with solution-driven adaptivity, we generalize problem (15) by introducing a dependency of $\mathscr{D}$ on the dual variable: find a fixed point $p^{*}$ of the mapping

$$
p_{0} \longmapsto \bar{p}:=\underset{p \in \mathscr{D}\left(p_{0}\right)}{\operatorname{argmin}} G(p)=F(p)+\Phi(p) .
$$

Having found a fixed point $p^{*}$, the corresponding constraint set is $\mathscr{D}\left(p^{*}\right)$; that is, the adaptivity is solution-driven. Solving problem (16) is equivalent to considering the following mixed quasi-variational inequality (MQVI):

$$
\text { find } \begin{aligned}
p^{*} \in \mathscr{D} & \left(p^{*}\right) \text { such that }\left\langle\nabla F\left(p^{*}\right), p-p^{*}\right\rangle \\
+ & +(p)-\Phi\left(p^{*}\right) \geq 0, \quad \forall p \in \mathscr{D}\left(p^{*}\right),
\end{aligned}
$$

where $F(p)=(1 / 2)\|A p-f\|_{2}^{2}$ and $\Phi(p)=\|A p\|_{2}$.

From (5), it is easy to see that the MQVI (17) is equivalent to the following projection equation:

$$
p^{*}=\Pi_{\mathscr{D}\left(p^{*}\right)}^{\Phi}\left(p^{*}-\rho \nabla F\left(p^{*}\right)\right), \quad \forall \rho>0 .
$$

\section{Theory for MQVI}

In this section, we provide the existence and uniqueness results for the MQVI (17).

\subsection{Existence of Solutions}

Theorem 2. Let $F(p):=(1 / 2)\|A p-f\|_{2}^{2}$ and $\Phi(p):=\|A p\|_{2}$, where $A: \mathbb{R}^{m n} \rightarrow \mathbb{R}^{n}$ is a linear operator. Let $\mathscr{D}(p)$ be defined as follows:

$$
\mathscr{D}: \bar{p} \rightrightarrows \mathscr{D}(\bar{p}):=\left\{p \in \mathbb{R}^{m n}: p_{i} \in \mathscr{D}_{l o c}^{i}(\bar{p}) \subset \mathbb{R}^{m n}, i=1, \ldots, n\right\},
$$

where each $\mathscr{D}_{\text {loc }}^{i}: \mathbb{R}^{m n} \rightrightarrows \mathbb{R}^{m n}, i=1, \ldots, n$, has the following properties:

(i) For fixed $p$ the set $\mathscr{D}_{l o c}^{i}(p)$ is a closed convex subset of $\mathbb{R}^{m n}$.

(ii) There exists $C>0$ such that, for all $i, p$, one has $\mathscr{D}_{l o c}^{i}(p) \subset B_{C}(0)$. 
(iii) There exists $c>0$ such that, for every $p$ and every $i$, one has $B_{c}(0) \subset \mathscr{D}_{l o c}^{i}(p)$. In particular, $\mathscr{D}_{\text {loc }}^{i}(p)$ is nonempty.

(iv) The generalized $\Phi$-projection operator $\Pi_{\mathscr{D}_{\text {loc }}^{i}(p)}^{\Phi}(q)$ of $q$ onto $\mathscr{D}_{\text {loc }}^{i}(p)$ for a fixed $q$ is continuous with respect to $p$.

Then mixed quasi-variational inequality (17) has a solution.

Proof. Firstly, we know from the definition of $\mathscr{D}(p)$ that

$$
\mathscr{D}(p):=\mathscr{D}_{\mathrm{loc}}^{1}(p) \times \mathscr{D}_{\mathrm{loc}}^{2}(p) \times \cdots \times \mathscr{D}_{\mathrm{loc}}^{n}(p) .
$$

Thus, from assumption (ii), we immediately derive that

$$
\mathscr{D}(p) \subset\left(B_{C}(0)\right)^{n} \subset B_{\sqrt{n} C}(0), \quad p \in \mathbb{R}^{m n} .
$$

Since $B_{\sqrt{n} C}(0)$ is a bounded closed convex ball, $B_{\sqrt{n} C}(0)$ is compact convex.

Assumptions (i) and (iii) imply that $\mathscr{D}$ is a nonempty closed convex valued mapping on $B_{\sqrt{n} C}(0)$. Moreover, $\Phi(p)=$ $\|A p\|_{2}$ is a proper, convex, and lower semicontinuous function. Hence the generalized $\Phi$-projection operator $\Pi_{\mathscr{D}(p)}^{\Phi}$ is well defined. By $\Pi_{\mathscr{D}(p)}^{\Phi}(q)=\left(\Pi_{\mathscr{D}_{\mathrm{loc}}^{1}(p)}^{\Phi}\left(q_{1}\right), \ldots, \Pi_{\mathscr{D}_{\mathrm{loc}}^{n}(p)}^{\Phi}\left(q_{n}\right)\right)^{\top}$ and (iv), we obtain that $\Pi_{\mathscr{D}(p)}^{\Phi}$ is continuous with respect to $p$.

Now we define the mapping $S: B_{\sqrt{n} C}(0) \rightarrow B_{\sqrt{n} C}(0)$ by

$$
S(p):=\Pi_{\mathscr{D}(p)}^{\Phi}(p-\rho \nabla F(p)), \quad \forall \rho>0 .
$$

It follows from the continuity of $\nabla F(p)=A^{\top}(A p-f)$ and $\Pi_{\mathscr{D}(p)}^{\Phi}$ that $S$ is continuous. Hence by (18) and the Brouwer fixed point theorem, we have that problem (17) has a solution.

In the case that $\Phi \equiv 0$, a similar result of Theorem 2 was obtained (see Proposition 4.2 of [19]). Therefore, Theorem 2 can also be considered as a generalization of Proposition 4.2 of [19].

5.2. Uniqueness Result of the Proposed Approach. In this subsection, let us consider the uniqueness results for MQVI (17). In [19], Lenzen et al. discussed the uniqueness of solution of quasi-variational inequality on only a subspace of $\mathbb{R}^{m n}$, because $\nabla F$ is not strongly monotone on the null space $\mathcal{N}(A)$ of $A\left(\mathcal{N}(A):=\left\{x \in \mathbb{R}^{n}: A x=0\right\}\right)$. On the other hand, our main aim is to find $u=M^{-1}(f-A p)$, which does not depend on the component of $p$ in $\mathscr{N}(A)$. In view of these reasons mentioned, [19] restricted the problem of quasi-variational inequality to the complement $\mathcal{N}^{\perp}(A)$ of $\mathcal{N}(A)$. Now we utilize similar method to study problem (17). Firstly, we give the following search model that is restricted to $\mathscr{N}^{\perp}(A)$ :

$$
\begin{aligned}
& \text { Find } p^{*} \in \Pi_{\mathcal{N}^{\perp}(A)} \mathscr{D}\left(p^{*}\right) \\
& \text { such that }\left\langle\nabla F\left(p^{*}\right), p-p^{*}\right\rangle+\Phi(p)-\Phi\left(p^{*}\right) \geq 0, \\
& \forall p \in \Pi_{\mathcal{N}^{\perp}(A)} \mathscr{D}\left(p^{*}\right) .
\end{aligned}
$$

Theorem 3. Assume that the set $\mathscr{D}(p)$ depends only on $p_{\text {res }}:=$ $\Pi_{\mathcal{N}^{\perp}(A)}(p)$.

(i) Let $p_{\text {res }}^{*}$ be a solution to the restricted problem (23), and then any $p^{*} \in \Pi_{\mathcal{N}^{\perp}(A)}^{-1}\left(p_{\text {res }}^{*}\right) \cap \mathscr{D}\left(p_{\text {res }}^{*}\right)$ is a solution to the original problem (17).

(ii) Let $p^{*}$ be a solution to the unrestricted problem (17), and then any $p_{\text {res }}^{*} \in \Pi_{\mathcal{N}^{\perp}(A)}^{-1}\left(p^{*}\right)$ is a solution to the restricted problem (23).

Proof. Let $p_{\text {res }}^{*} \in \Pi_{\mathcal{N}^{\perp}(A)}\left(\mathscr{D}\left(p_{\text {res }}^{*}\right)\right)$ be a solution to the restricted problem (23); that is,

$$
\begin{aligned}
&\left\langle\nabla F\left(p_{\text {res }}^{*}\right), p-p_{\text {res }}^{*}\right\rangle+\Phi(p)-\Phi\left(p_{\text {res }}^{*}\right) \geq 0, \\
& \forall p \in \Pi_{\mathcal{N}^{\perp}(A)}\left(\mathscr{D}\left(p_{\text {res }}^{*}\right)\right) .
\end{aligned}
$$

For any $p^{*} \in \mathscr{D}\left(p_{\text {res }}^{*}\right)$ such that $p_{\text {res }}^{*}=\Pi_{\mathcal{N}^{\perp}(A)} p^{*}=p^{*}-$ $\Pi_{\mathscr{N}(A)} p^{*}$, it holds that $p^{*} \in \mathscr{D}\left(p^{*}\right)=\mathscr{D}\left(p_{\text {res }}^{*}\right)$. Now let $p \in$ $\mathscr{D}\left(p_{\text {res }}^{*}\right)=\mathscr{D}\left(p^{*}\right)$ be arbitrary. We decompose $p$ into $p=$ $p_{\text {res }}+p_{\mathcal{N}}$, where $p_{\text {res }}:=\Pi_{\mathcal{N}^{\perp}(A)} p$ and $p_{\mathcal{N}}:=\Pi_{\mathcal{N}(A)} p$. Then it follows from (25) and $A p=A p_{\text {res }}$ and $A p^{*}=A p_{\text {res }}^{*}$ that

$$
\begin{aligned}
\langle\nabla F & \left.\left(p^{*}\right), p-p^{*}\right\rangle+\Phi(p)-\Phi\left(p^{*}\right) \\
= & \left\langle A^{\top}\left(A p^{*}-f\right), p-p^{*}\right\rangle+\|A p\|_{2}-\left\|A p^{*}\right\|_{2} \\
= & \left\langle A p^{*}-f, A\left(p-p^{*}\right)\right\rangle+\|A p\|_{2}-\left\|A p^{*}\right\|_{2} \\
= & \left\langle A p_{\text {res }}^{*}-f, A\left(p_{\text {res }}-p_{\text {res }}^{*}\right)\right\rangle+\left\|A p_{\text {res }}\right\|_{2} \\
& -\left\|A p_{\text {res }}^{*}\right\|_{2} \\
= & \left\langle A^{\top}\left(A p_{\text {res }}^{*}-f\right), p_{\text {res }}-p_{\text {res }}^{*}\right\rangle+\left\|A p_{\text {res }}\right\|_{2} \\
& -\left\|A p_{\text {res }}^{*}\right\|_{2} \geq 0 .
\end{aligned}
$$

Thus, $p^{*}$ is a solution of (17).

Let $p^{*}$ be a solution of problem (17). In particular, $p^{*} \epsilon$ $\mathscr{D}\left(p^{*}\right)$. We consider the decomposition $p^{*}=p_{\text {res }}^{*}+p_{\mathcal{N}}^{*}$, where $p_{\text {res }}^{*}:=\Pi_{\mathcal{N}^{\perp}(A)} p^{*}$ and $p_{\mathcal{N}}^{*}:=\Pi_{\mathcal{N}(A)} p^{*}$. Then

$$
p_{\text {res }}^{*} \in \Pi_{\mathcal{N}^{\perp}(A)}\left(\mathscr{D}\left(p^{*}\right)\right)=\Pi_{\mathcal{N}^{\perp}(A)}\left(\mathscr{D}\left(p_{\text {res }}^{*}\right)\right) .
$$

Let $p_{\text {res }} \in \Pi_{\mathcal{N}^{\perp}(A)}\left(\mathscr{D}\left(p_{\text {res }}^{*}\right)\right)$ be arbitrary. There exists $p \in$ $\mathscr{D}\left(p_{\text {res }}^{*}\right)$ such that

$$
p_{\text {res }}=\Pi_{\mathcal{N}^{\perp}(A)} p=p-\Pi_{\mathcal{N}(A)} p .
$$

It follows from (28) that

$$
\begin{aligned}
\langle\nabla F & \left.\left(p_{\text {res }}^{*}\right), p_{\text {res }}-p_{\text {res }}^{*}\right\rangle+\Phi\left(p_{\text {res }}\right)-\Phi\left(p_{\text {res }}^{*}\right) \\
= & \left\langle A p_{\text {res }}^{*}-f, A\left(p_{\text {res }}-p_{\text {res }}^{*}\right)\right\rangle+\left\|A p_{\text {res }}\right\|_{2} \\
& -\left\|A p_{\text {res }}^{*}\right\|_{2} \\
= & \left\langle A p^{*}-f, A\left(p-p^{*}\right)\right\rangle+\|A p\|_{2}-\left\|A p^{*}\right\|_{2} \\
= & \left\langle A^{\top}\left(A p^{*}-f\right), p-p^{*}\right\rangle+\|A p\|_{2}-\left\|A p^{*}\right\|_{2} \\
\geq & 0,
\end{aligned}
$$

where the last inequality holds since $p \in \mathscr{D}\left(p^{*}\right)$ due to $\mathscr{D}\left(p^{*}\right)=\mathscr{D}\left(p_{\text {res }}^{*}\right)$ and $p^{*}$ solves (17). Thus $p_{\text {res }}^{*}$ is a solution of (23). 
From Section 3, we can see that the final purpose of finding the existence and uniqueness of $p^{*}$ is to solve the optimal problem (10), because $u^{*}=M^{-1}\left(f-A p^{*}\right)$ which does not depend on $p_{N}^{*}$. We therefore focus on $v^{*}=A p^{*}$, which depends only on the component $p_{\text {res }}^{*}$ of $p^{*}$ in $\mathscr{N}^{\perp}(A)$; that is, we only need to consider the restricted problem (23). Based on Theorem 3, the restricted problem has a solution if and only if the original problem has a solution. Here we specify $\left\|v^{*}\right\|_{2}=\left\|p^{*}\right\|_{B}:=\sqrt{\left(p^{*}\right)^{\top} A^{\top} A p^{*}}$. In the following discussion, $\widetilde{D}(v)$ denotes a nonempty, closed, convex set such that $\widetilde{\mathscr{D}}(v)=\mathscr{D}(p)=\widetilde{\mathscr{D}}(A p)$.

Before showing the uniqueness, let us define $T: \mathbb{R}^{n} \rightrightarrows$ $\mathbb{R}^{m n}$ such that $\bar{p} \in T(v)$ if and only if $\bar{p} \in \widetilde{\mathscr{D}}(v)$ and $\bar{p}$ is a solution to the following MQVI:

$$
\langle\nabla F(\bar{p}), p-\bar{p}\rangle+\Phi(p)-\Phi(\bar{p}) \geq 0, \quad \forall p \in \widetilde{D}(v) .
$$

Theorem 4. Under all the assumption conditions of Theorem 2 and the assumptions

(i) $\nabla F$ is Lipschitz continuous with Lipschitz constant $\mu_{2}:=$ $\left\|A^{\top} A\right\|_{2}>0$, that is,

$\|\nabla F(x)-\nabla F(y)\|_{2} \leq \mu_{2}\|x-y\|_{2}, \quad \forall x, y \in \mathbb{R}^{m n}$,

(ii) the generalized $\Phi$-projection operator $\Pi_{\mathscr{D}(v)}^{\Phi} q$ is Lipschitz continuous with respect to $v$ with the variation rate $\tilde{\eta}>0$, that is,

$$
\left\|\Pi_{\mathscr{D}(v)}^{\Phi} q-\Pi_{\mathscr{D}\left(v^{\prime}\right)}^{\Phi} q\right\|_{2} \leq \tilde{\eta}\left\|v-v^{\prime}\right\|_{2}, \quad \forall q \in \mathbb{R}^{m n},
$$

(iii) $\tilde{\eta}<1 / \sqrt{\mu_{2}}$,

if $p^{*}$ is a solution of mixed quasi-variational inequality (17), then $v^{*}=A p^{*}$ is unique.

Proof. Fix $v_{1}, v_{2} \in \operatorname{Im}(A)$. Let $\mathscr{D}_{i}:=\widetilde{\mathscr{D}}\left(v_{i}\right), p_{i} \in T\left(v_{i}\right)$ :

(i) If $A\left(p_{1}-p_{2}\right)=0$, we obtain

$$
\begin{aligned}
& \left\|A T\left(v_{1}\right)-A T\left(v_{2}\right)\right\|_{2}=\left\|A p_{1}-A p_{2}\right\|_{2}=0 \\
& \quad<\left\|v_{1}-v_{2}\right\|_{2} .
\end{aligned}
$$

(ii) If $A\left(p_{1}-p_{2}\right) \neq 0$, since $p_{i}, i=1,2$, solve $\arg \min _{p \in \widetilde{\mathscr{D}}\left(v_{i}\right)}(1 / 2)\|A p-f\|_{2}^{2}+\|A p\|_{2}$, the following MQVI

$$
\left\langle\nabla F\left(p_{i}\right), q-p_{i}\right\rangle+\Phi(q)-\Phi\left(p_{i}\right) \geq 0, \quad \forall q \in \mathscr{D}_{i},
$$

holds; that is, for any $\rho \geq 0$,

$$
p_{i}=\Pi_{\mathscr{D}_{i}}^{\Phi}\left(p_{i}-\rho \nabla F\left(p_{i}\right)\right) .
$$

In particular, $q:=\Pi_{\mathscr{D}_{2}}^{\Phi}\left(p_{1}-\rho \nabla F\left(p_{1}\right)\right)$, and it follows from (35) and (ii) that

$$
\begin{aligned}
& \left\|p_{1}-q\right\|_{2} \\
& \quad=\left\|\Pi_{\mathscr{D}_{1}}^{\Phi}\left(p_{1}-\rho \nabla F\left(p_{1}\right)\right)-\Pi_{\mathscr{D}_{2}}^{\Phi}\left(p_{1}-\rho \nabla F\left(p_{1}\right)\right)\right\|_{2} \\
& \quad \leq \tilde{\eta}\left\|v_{1}-v_{2}\right\|_{2} .
\end{aligned}
$$

On the other hand, $p_{2} \in \mathscr{D}_{2}$ implies that

$$
\left\langle q-\left(p_{1}-\rho \nabla F\left(p_{1}\right)\right), p_{2}-q\right\rangle+\Phi\left(p_{2}\right)-\Phi(q) \geq 0 .
$$

Therefore, from (34) and (37), we have

$$
\begin{aligned}
\left\langle q-p_{1}, p_{2}-q\right\rangle \geq & \rho\left\langle\nabla F\left(p_{1}\right), q-p_{2}\right\rangle-\Phi\left(p_{2}\right) \\
& +\Phi(q) \\
= & \rho\left\langle\nabla F\left(p_{1}\right), q-p_{1}\right\rangle \\
& +\rho\left\langle\nabla F\left(p_{2}\right), p_{1}-q\right\rangle \\
& +\rho\left\langle\nabla F\left(p_{2}\right), q-p_{2}\right\rangle+\Phi(q) \\
& -\Phi\left(p_{2}\right) \\
& +\rho\left\langle\nabla F\left(p_{1}\right)-\nabla F\left(p_{2}\right), p_{1}-p_{2}\right\rangle \\
\geq & \rho\left\langle\nabla F\left(p_{1}\right)-\nabla F\left(p_{2}\right), q-p_{1}\right\rangle \\
& +\rho\left\langle\nabla F\left(p_{1}\right)-\nabla F\left(p_{2}\right), p_{1}-p_{2}\right\rangle \\
= & \rho\left\langle A^{\top} A\left(p_{1}-p_{2}\right), q-p_{1}\right\rangle \\
& +\rho\left\|A p_{1}-A p_{2}\right\|_{2}^{2} ;
\end{aligned}
$$

that is,

$$
\begin{aligned}
\rho\left\|A p_{1}-A p_{2}\right\|_{2}^{2} \leq & \left\langle q-p_{1}, p_{2}-q\right\rangle \\
& +\rho\left\langle A^{\top} A\left(p_{1}-p_{2}\right), p_{1}-q\right\rangle \\
\leq & \left\|q-p_{1}\right\|_{2} \cdot\left\|q-p_{2}\right\|_{2} \\
& +\rho\left\|A p_{1}-A p_{2}\right\|_{2} \cdot\left\|A p_{1}-A q\right\|_{2}
\end{aligned}
$$

and, by dividing by $\rho\left\|A p_{1}-A p_{2}\right\|_{2}>0$, because $A p_{1}-A p_{2} \neq 0$,

$$
\begin{aligned}
\left\|A p_{1}-A p_{2}\right\|_{2} \leq & \frac{\left\|q-p_{1}\right\|_{2} \cdot\left\|q-p_{2}\right\|_{2}}{\rho\left\|A p_{1}-A p_{2}\right\|_{2}} \\
& +\left\|A p_{1}-A q\right\|_{2} .
\end{aligned}
$$

Since $\rho$ is arbitrarily large, we find

$$
\left\|A p_{1}-A p_{2}\right\|_{2} \leq\left\|A p_{1}-A q\right\|_{2} .
$$

By (i), we have $\|A\|_{2}=\sqrt{\mu_{2}}$. Thus, it follows from (36) that

$$
\left\|A p_{1}-A q\right\|_{2} \leq\|A\|_{2}\left\|p_{1}-q\right\|_{2} \leq \sqrt{\mu_{2}} \tilde{\eta}\left\|v_{1}-v_{2}\right\|_{2} ;
$$

that is,

$$
\left\|A T\left(v_{1}\right)-A T\left(v_{2}\right)\right\|_{2} \leq \sqrt{\mu_{2}} \widetilde{\eta}\left\|v_{1}-v_{2}\right\|_{2} .
$$

Therefore, we can see from (iii) that $A \circ T: \mathbb{R}^{n} \rightarrow \mathbb{R}^{n}$ is a contractive mapping. Moreover, the Banach fixed point theorem implies that there exists a unique fixed point $v^{*}=$ $A p^{*}$ of $A \circ T$ in $\mathbb{R}^{n}$.

We already mentioned that, in the considered applications for image restoration, we are actually interested in the variable $u:=M^{-1}(f-A p)$. Obviously, it follows from Theorem 4 that $u$ is unique. 
Input: The maximal number of iterations $N$; the constraint set $\mathscr{D}\left(p^{0}\right)$; the starting point $p^{0} \in \mathscr{D}\left(p^{0}\right) \subset B_{\sqrt{n} C}(0)$; the parameter number $\rho$ and such that $0<\rho<1 / \mu_{2}$.

Output: $p^{N}$.

begin

for $k=0$ to $N-1$ do

Step 1. $\bar{p}^{k-1}:=\Pi_{\mathscr{D}\left(p^{k-1}\right)}^{\Phi}\left(p^{k-1}-\rho \nabla F\left(p^{k-1}\right)\right)$;

Step 2. $p^{k}:=\Pi_{\mathscr{D}\left(\bar{p}^{k-1}\right)}^{\Phi}\left(p^{k-1}-\rho \nabla F\left(\bar{p}^{k-1}\right)\right)$; end

Step 3. If $\left|p^{k}-p^{k-1}\right| \leq \varepsilon$, for all $\varepsilon>0$, then, stop; otherwise, $k=k+1$ and go the Step 1 .

end

Algorithm 1: Modified projection method for solving the MQVI (17).

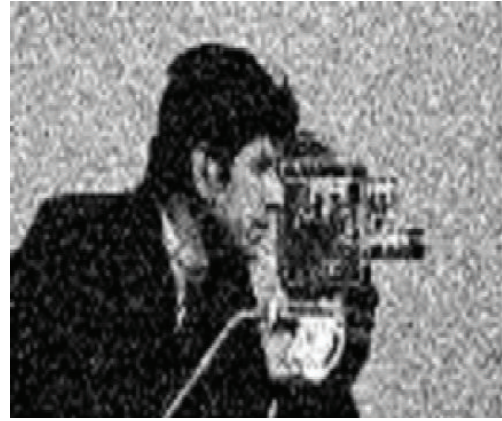

(a)

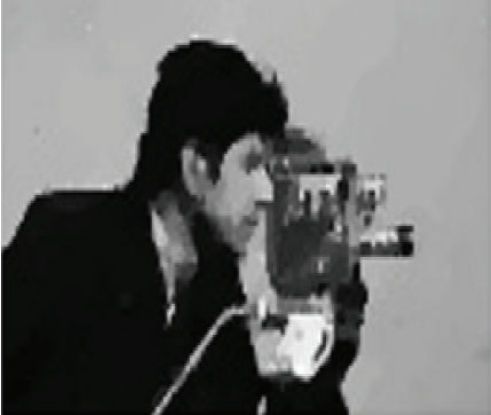

(b)

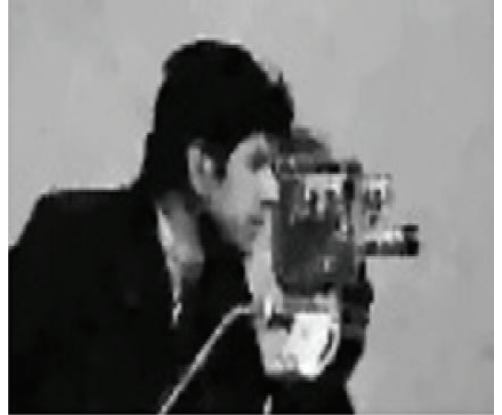

(c)

FIGURE 1: (a) Noisy input data, MSSIM = 0.327. (b) Solution-driven adaptive TV, MSSIM $=0.812$. (c) Solution-driven adaptive generalized TV, MSSIM $=0.822$.

\section{Numerics}

Throughout this section, assume that all the assumption conditions of Theorems 2 and 4 are satisfied. Next, we propose an iterative algorithm to solve the MQVI (17) and show convergence of the proposed algorithm.

\subsection{Proposed Iterative Algorithm. See Algorithm 1.}

\subsection{Convergence of Algorithm 1}

Theorem 5. Let all the assumptions of Theorems 2 and 4 be satisfied. Moreover, assume that $\nabla F$ is monotone; that is,

$$
\langle\nabla F(x)-\nabla F(y), x-y\rangle \geq 0, \quad \forall x, y \in \mathbb{R}^{m n} .
$$

Then the sequence $\left\{p^{k}\right\}$ generated by Algorithm 1 converges to the unique solution $p^{*}$ of MQVI (17).

Proof. For the proof, see Theorem 2.5 in [26].

\section{Experiment Results}

7.1. Improvement of Solution-Driven Adaptive TV Regularization. In this section, we show that the adaptivity is improved by switching from a solution-driven adaptive TV regularization to a solution-driven adaptive generalized TV model. To this end, we consider the image Cameraman. We generate test data for the denoising problem by adding Gaussian noise with zero mean and standard deviation 0.1 and for the deblurring problem by applying a blurring operator and adding Gaussian noise with zero mean and standard deviation 0.01. For comparison, we make use of a mean SSIM (MSSIM) index [27] to evaluate the restored image quality in Figures 1 and 2. The definitions of the similarity measures SSIM and MSSIM are given as follows:

$$
\operatorname{SSIM}(x, y)=\frac{\left(2 \mu_{x} \mu_{y}+c_{1}\right)\left(2 \sigma_{x} \sigma_{y}+c_{2}\right)}{\left(\mu_{x}^{2}+\mu_{y}^{2}+c_{1}\right)\left(\sigma_{x}^{2}+\sigma_{y}^{2}+c_{2}\right)}
$$

where $\mu_{x}, \mu_{y}$ are the average values of two signals $x$ and $y, \sigma_{x}^{2}$ and $\sigma_{y}^{2}$ are the variances of $x$ and $y$, and $c_{1}, c_{2}$ are variables to stabilize the division with weak denominator

$$
\operatorname{MSSIM}(X, Y)=\frac{1}{M} \sum_{j=1}^{M} \operatorname{SSIM}\left(x_{j}, y_{j}\right),
$$

where $X$ and $Y$ are the reference and the distorted images, respectively; $x_{j}$ and $y_{j}$ are the image contents at the $j$ th local window; and $M$ is the number of local windows of the image.

In Figure 1, we give the close-up of the results of denoising the image Cameraman with TV regularization and generalized TV regularization approaches. The values 


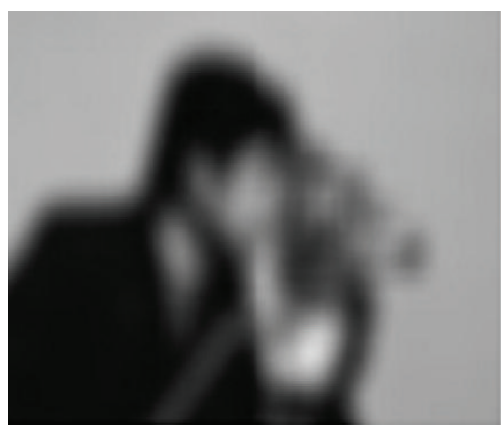

(a)

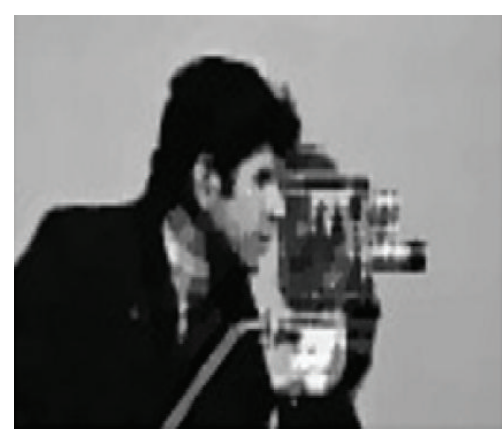

(b)

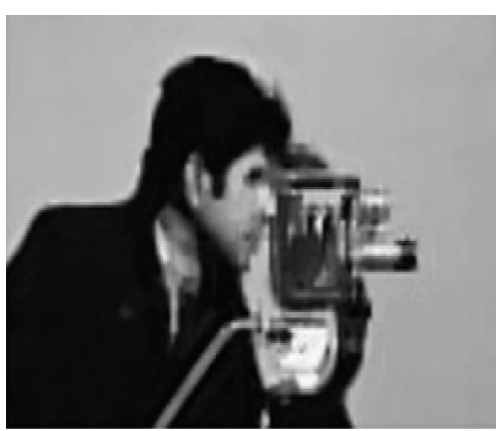

(c)

FIGURE 2: (a) Blurred input data, MSSIM = 0.032. (b) Solution-driven adaptive TV, MSSIM $=0.866$. (c) Solution-driven adaptive generalized TV, MSSIM $=0.868$.

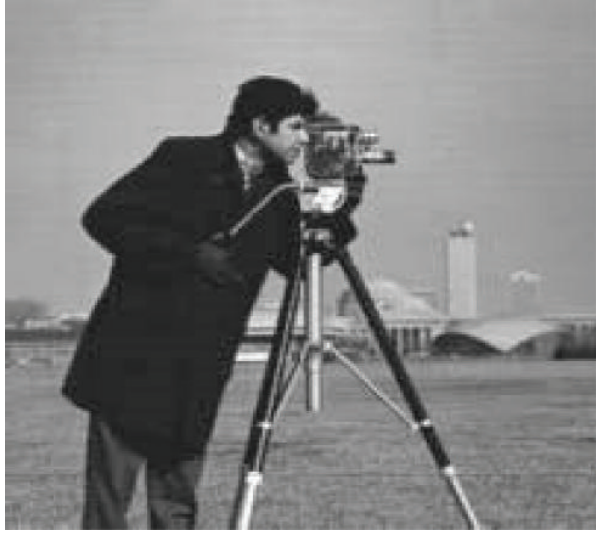

(a)

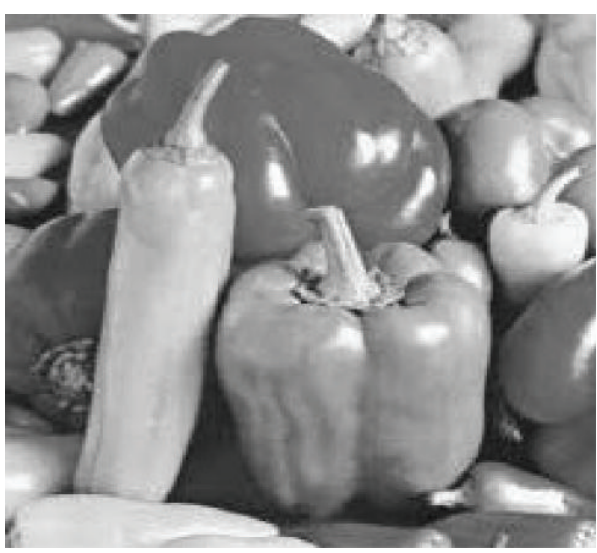

(c)

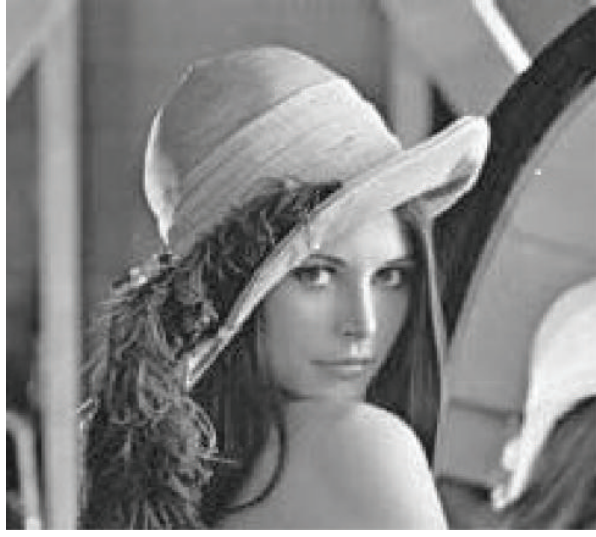

(b)

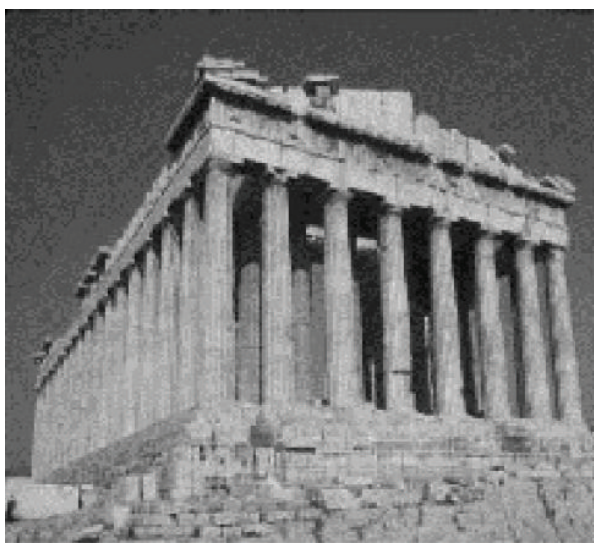

(d)

Figure 3: Test images. (a) Cameraman. (b) Lena. (c) Pepper. (d) Building.

for similarity given show that generalized TV regularization approach enhances the reconstruction compared to the TV regularization.

In Figure 2, close-up of the results of deblurring the image Cameraman with TV regularization and generalized TV regularization approaches is shown. In terms of similarity to the original data, we can see that generalized TV regularization approach improves the reconstruction compared to the TV regularization.

7.2. Comparison of Image Restoration for Different Test Images. In this subsection, we report that our method can remove Gaussian noise efficiently while preserving details very well. For noise removal, we compare our method with some 


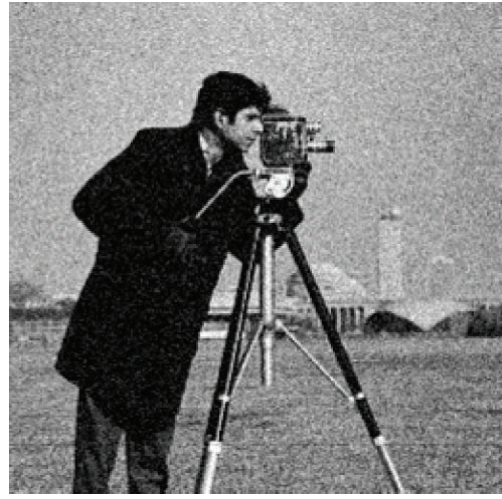

(a)

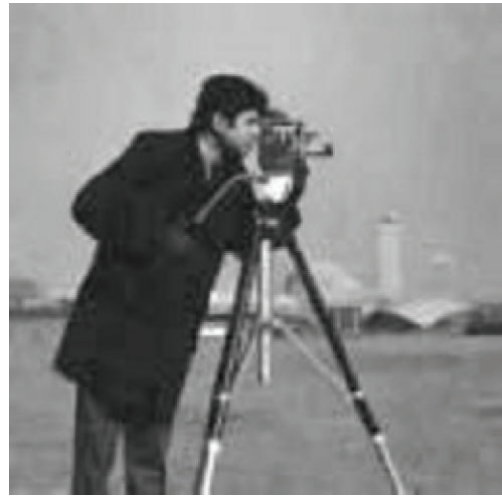

(d)

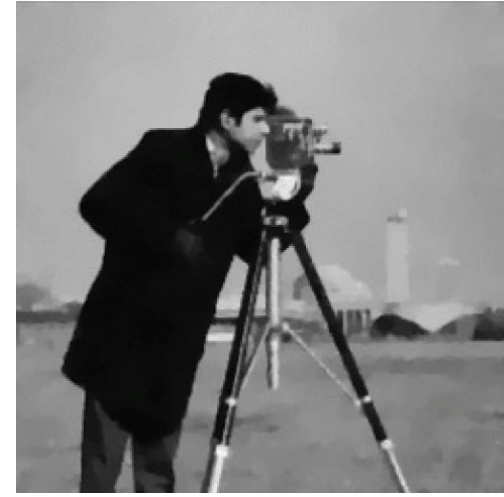

(b)

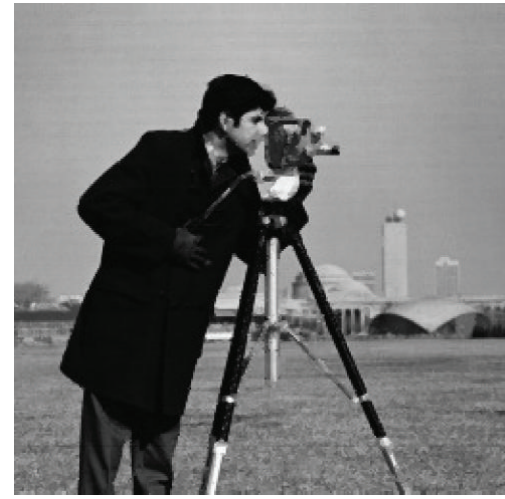

(e)

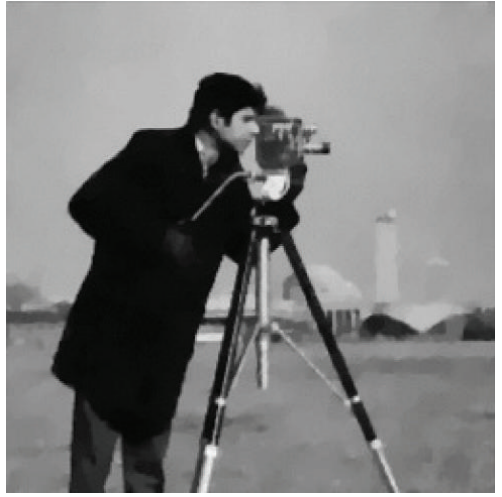

(c)

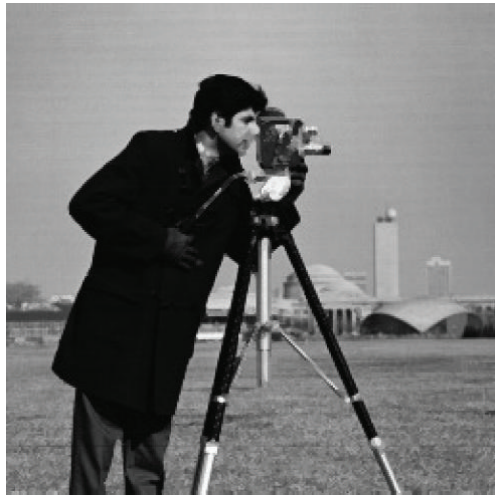

(f)

Figure 4: (a) Noisy image (PSNR = $8.748 \mathrm{~dB})$. (b) Local TV, Split Bregman (PSNR = $28.18 \mathrm{~dB})$. (c) Nonlocal TV, Split Bregman $($ PSNR $=$ $30.35 \mathrm{~dB}$ ). (d) Anisotropic TV, Split Bregman (PSNR = 27.687 dB). (e) Isotropic TV, Split Bregman (PSNR = 28.594 dB). (f) Solution-driven adaptive generalized TV, modified projection $(\mathrm{PSNR}=30.56 \mathrm{~dB})$

recently developed models, namely, the local TV [28], the NLTV [29], the anisotropic TV [30], and the isotropic TV [30], when the Split Bregman method is considered. The quality of our restoration is measured by the peak signal-tonoise ratio (PSNR) in decibels $(\mathrm{dB})$ :

$$
\operatorname{PSNR}=10 \log _{10}\left(\frac{M \times N}{\sum_{i=1}^{M} \sum_{j=1}^{N}\left(u_{i, j}-\widetilde{u}_{i, j}\right)^{2}}\right),
$$

where $M \times N$ indicates the image size and $u$ and $\tilde{u}$ denote the original image and the restored one, respectively. Generally, the higher PSNR values indicate better quality of the restored images.

The test images are shown in Figure 3.

In Figure 4, we make use of the local TV model, the NLTV model, the anisotropic TV model, the isotropic TV model, and the solution-driven adaptive generalized TV model to display the restoration results for the test image (a) corrupted by $8 \%$ Gaussian noise. Here, our proposed model performs significantly better and can suppress the Gaussian noise successfully.

Table 1 lists the restoration results in the PSNR of different methods for test images Cameraman, Lena, Pepper, and Building corrupted by Gaussian noise.
TABLE 1: Comparison of restoration results in PSNR (dB) for images corrupted by Gaussian noise.

\begin{tabular}{lcccc}
\hline Methods & Cameraman & Lena & Pepper & Building \\
\hline Local TV & 28.18 & 27.68 & 26.68 & 27.55 \\
NLTV & 30.35 & 29.87 & 30.86 & 29.23 \\
Anisotropic TV & 27.687 & 26.1 & 26.591 & 29.3 \\
Isotropic TV & 28.594 & 27.902 & 27.661 & 26.603 \\
Proposed & $\mathbf{3 0 . 5 6}$ & $\mathbf{3 0 . 2 1}$ & $\mathbf{3 0 . 9 8}$ & $\mathbf{2 9 . 5}$ \\
\hline
\end{tabular}

From Table 1, it is apparent that our proposed method generates the best restoration results for all the test images. Actually, in Table 1, the proposed method obtains the highest PSNR values for all the test images. This demonstrated that our method is more robust for images corrupted by Gaussian noise.

7.3. Expanded Experiments. Remote sensing very often deals with inverting a forward model. To this aim, one has to produce an accurate and robust model able to predict physical, chemical, geological, or atmospheric parameters from spectra, such as surface temperature, water vapour, and ozone; see, for example, [31]. Denoising images could be part 


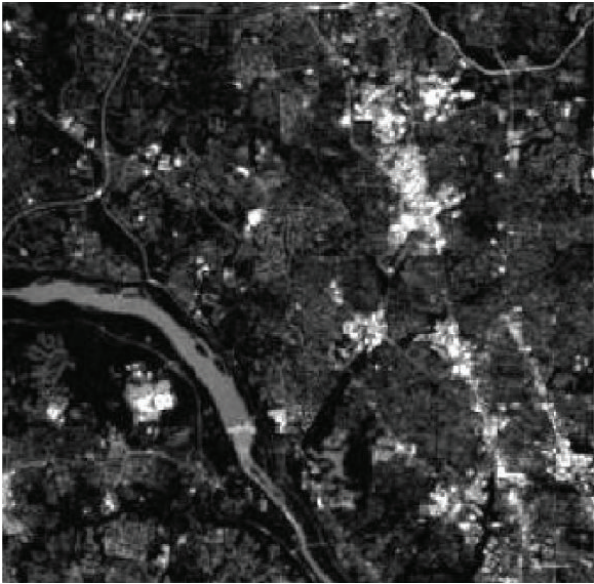

(a)

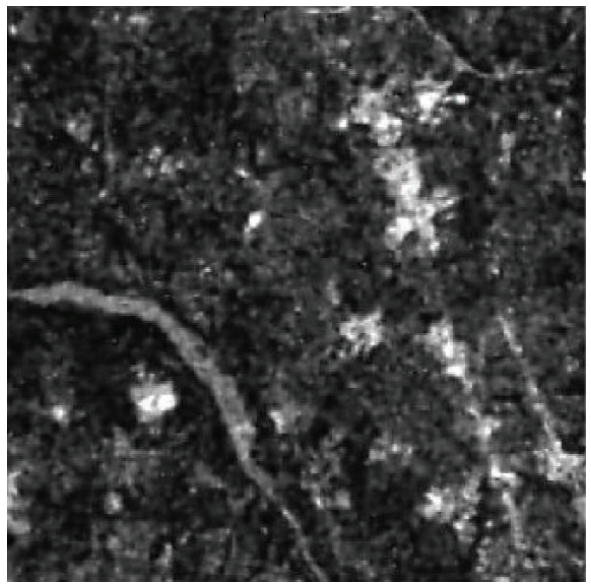

(c)

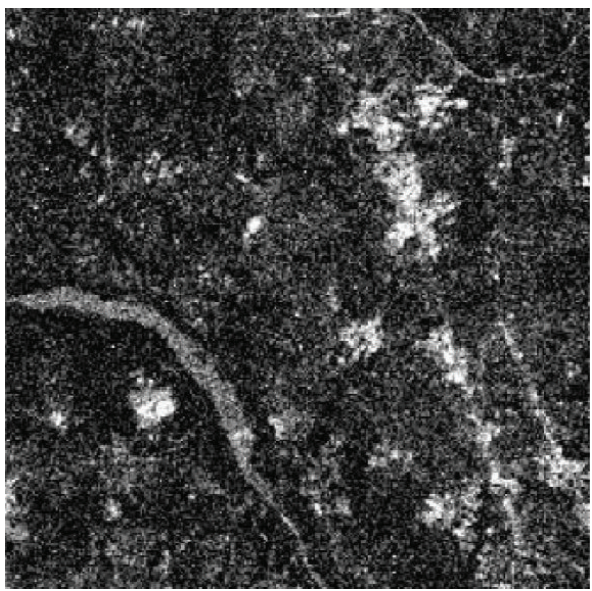

(b)

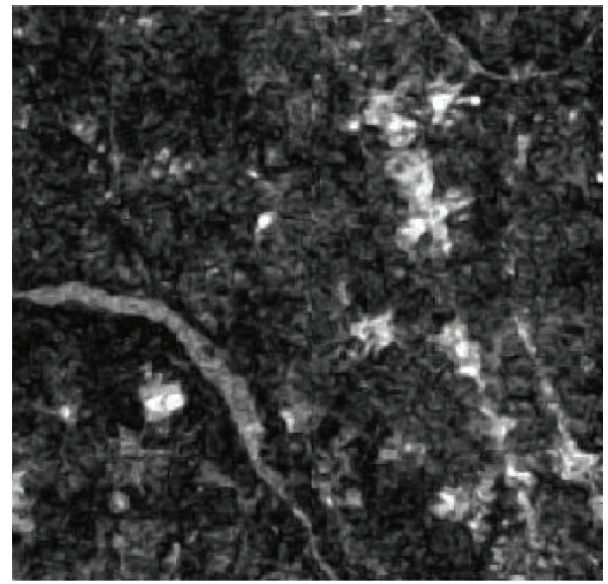

(d)

FIGURE 5: (a) Original satellite image. (b) Gaussian noise $(\sigma=0.02)$ image. (c) Fourth-order PDE model. (d) Proposed method.

of the accurate and robust model. In particular, our image restoration method could be used to remove noises in the class of satellite images.

In Figure 5, we give the close-up of the results of denoising the satellite image adding Gaussian noise with the standard derivation $\sigma=0.02$. Though the fourth-order PDE model removes noise more thoroughly, texture detail also had been changed. Comparing rivers and streets with those in the original image, some information was lost. Our method not only suppresses the noise very well but also keeps a lot of detail and texture information of the original image.

From Table 2, we can see that our method has better denoising effects than pure anisotropic diffusion and fourthorder PDE.

\section{Conclusion}

In this paper, our main contribution is to introduce the generalized TV regularization which includes various types of TV regularization as special cases. We studied the existence and uniqueness of the solution for mixed quasi-variational
TABLE 2: Comparison of restoration results in PSNR (dB) for satellite images.

\begin{tabular}{lcccc}
\hline Methods & $\sigma=0.01$ & $\sigma=0.02$ & $\sigma=0.05$ & $\sigma=1$ \\
\hline Pure anisotropic diffusion [23] & 30.16 & 23.68 & 15.36 & 6.10 \\
Fourth-order PDE [24] & 31.64 & 25.40 & 17.40 & 11.36 \\
Proposed & $\mathbf{3 4 . 3 2}$ & $\mathbf{2 7 . 6 8}$ & $\mathbf{1 9 . 5 4}$ & $\mathbf{1 3 . 2 8}$ \\
\hline
\end{tabular}

inequality for solution-driven adaptive image denoising and image deblurring with generalized TV regularization. The convergence of proposed algorithm for MQVI was proposed. Moreover, our experimental results showed that we improve the image restoration quality and apply the proposed model to deal with the images in many different fields. Our further work will consider utilizing the theory of other variational inequalities for solving optimization problems with other kinds of generalized TV regularizations.

\section{Competing Interests}

The authors declare that they have no competing interests. 


\section{Acknowledgments}

This work was supported by National Natural Science Foundation of China (Grants 61379019 and 11401493), Innovation Team Funds of Southwest University for Nationalities (Grant 14CXTD03), Innovative Research Team of the Education Department of Sichuan Province (Grant 15TD0050), The Science and Technology Department of Sichuan Province (Grant 2015JY0027), and Fundamental Research Funds for the Central Universities, Southwest University for Nationalities (Grant 2014NZYQN29).

\section{References}

[1] L. I. Rudin, S. Osher, and E. Fatemi, "Nonlinear total variation based noise removal algorithms," Physica D: Nonlinear Phenomena, vol. 60, no. 1-4, pp. 259-268, 1992.

[2] J.-F. Aujol, G. Gilboa, T. Chan, and S. Osher, "Structure-texture image decomposition-modeling, algorithms, and parameter selection," International Journal of Computer Vision, vol. 67, no. 1, pp. 111-136, 2006.

[3] I. Bayram and M. E. Kamasak, "A directional total variation," in Proceedings of the 20th European Signal Processing Conference (EUSIPCO '12), pp. 265-269, IEEE, Piscataway, NJ, USA, August 2012.

[4] B. Berkels, M. Burger, M. Droske, O. Nemitz, and M. Rumpf, "Cartoon extraction based on anisotropic image classification," in Vision, Modeling, and Visualization Proceedings, pp. 293-300, IOS, Amsterdam, The Netherlands, 2006.

[5] A. Buades, B. Coll, and J. M. Morel, "A review of image denoising algorithms, with a new one," Multiscale Modeling \& Simulation. A SIAM Interdisciplinary Journal, vol. 4, no. 2, pp. 490-530, 2005.

[6] G. Gilboa and S. Osher, "Nonlocal operators with applications to image processing," Multiscale Modeling \& Simulation, vol. 7, no. 3, pp. 1005-1028, 2008.

[7] K. Bredies, K. Kunisch, and T. Pock, "Total generalized variation,” SIAM Journal on Imaging Sciences, vol. 3, no. 3, pp. 492$526,2010$.

[8] S. Setzer, G. Steidl, and T. Teuber, "Infimal convolution regularizations with discrete l1-type functionals," Communications in Mathematical Sciences, vol. 9, no. 3, pp. 797-827, 2011.

[9] F. Lenzen, F. Becker, and J. Lellmann, "Adaptive second-order total variation: an approach aware of slope discontinuities," in Scale Space and Variational Methods in Computer Vision: 4th International Conference, SSVM 2013, Schloss Seggau, Leibnitz, Austria, June 2-6, 2013. Proceedings, vol. 7893 of Lecture Notes in Computer Science, pp. 61-73, Springer, New York, NY, USA, 2013.

[10] A. Chambolle, "An algorithm for total variation minimization and applications," Journal of Mathematical Imaging and Vision, vol. 20, no. 1-2, pp. 89-97, 2004.

[11] T. Chan, S. Esedoglu, F. Park, and A. Yip, “Total variation image restoration: overview and recent developments," in Handbook of Mathematical Models in Computer Vision, N. Paragios, Y. Chen, and O. D. Faugeras, Eds., vol. 17, chapter 2, pp. 17-31, Springer, New York, NY, USA, 2006.
[12] J. P. Oliveira, J. M. Bioucas-Dias, and M. A. T. Figueiredo, "Adaptive total variation image deblurring: a majorizationminimization approach," Signal Processing, vol. 89, no. 9, pp. 1683-1693, 2009.

[13] F. Facchinei and J.-S. Pang, Finite-Dimensional Variational Inequalities and Complementarity Problems, Springer, New York, NY, USA, 2003.

[14] X.-P. Luo, "Tikhonov regularization methods for inverse variational inequalities," Optimization Letters, vol. 8, no. 3, pp. 877$887,2014$.

[15] X.-P. Luo and J. Yang, "Regularization and iterative methods for monotone inverse variational inequalities," Optimization Letters, vol. 8, no. 4, pp. 1261-1272, 2014.

[16] X.-p. Luo and H. Yang, "Regularization and iterative methods for inverse mixed variational inequalities," Nonlinear Analysis Forum, vol. 19, pp. 53-63, 2014.

[17] X.-P. Luo and H. Yang, "Tikhonov regularization methods for inverse mixed variational inequalities," Advances in Nonlinear Variational Inequalities, vol. 17, no. 2, pp. 13-25, 2014.

[18] D. Chan and J.-S. Pang, "The generalized quasi-variational inequality problem," Mathematics of Operations Research, vol. 7, no. 2, pp. 211-222, 1982.

[19] F. Lenzen, J. Lellmann, F. Becker, and C. Schnörr, "Solving quasi-variational inequalities for image restoration with adaptive constraint sets," SIAM Journal on Imaging Sciences, vol. 7, no. 4, pp. 2139-2174, 2014.

[20] F. Lenzen, F. Becker, J. Lellmann, S. Petra, and C. Schnörr, "A class of quasi-variational inequalities for adaptive image denoising and decomposition," Computational Optimization and Applications, vol. 54, no. 2, pp. 371-398, 2013.

[21] F. Lenzen, F. Becker, J. Lellmann, S. Petra, and C. Schnörr, "Variational image denoising with adaptive constraint sets," in Scale Space and Variational Methods in Computer Vision: Third International Conference, SSVM 2011, Ein-Gedi, Israel, May 29June 2, 2011, Revised Selected Papers, vol. 6667 of Lecture Notes in Computer Science, pp. 206-217, Springer, New York, NY, USA, 2012.

[22] M. A. Noor and K. I. Noor, "Some new classes of extended general mixed quasi-variational inequalities," Abstract and Applied Analysis, vol. 2012, Article ID 962978, 9 pages, 2012.

[23] L. Alvarez and J. M. Morel, "Formalization and computational aspects of image analysis," Acta Numerica, vol. 3, pp. 1-59, 1994.

[24] Y.-L. You and M. Kaveh, "Fourth-order partial differential equations for noise removal," IEEE Transactions on Image Processing, vol. 9, no. 10, pp. 1723-1730, 2000.

[25] X. Li, X. S. Li, and N. J. Huang, "A generalized f-projection algorithm for inverse mixed variational inequalities," Optimization Letters, vol. 8, no. 3, pp. 1063-1076, 2014.

[26] A. Nagurney, Network Economics. A Variational Inequality Approach, Kluwer Academics Publishers, Boston, Mass, USA, 1999.

[27] Z. Wang, A. C. Bovik, H. R. Sheikh, and E. P. Simoncelli, "Image quality assessment: from error visibility to structural similarity," IEEE Transactions on Image Processing, vol. 13, no. 4, pp. 600612, 2004.

[28] T. Goldstein and S. Osher, "The split Bregman algorithm for L1 regularized problems," SIAM Journal on Imaging Sciences, vol. 2, no. 2, pp. 323-343, 2009. 
[29] X. W. Liu and L. H. Huang, "A new nonlocal total variation regularization algorithm for image denoising," Mathematics and Computers in Simulation, vol. 97, pp. 224-233, 2014.

[30] Z. W. Qin, D. Goldfarb, and S. Q. Ma, "An alternating direction method for total variation denoising," Optimization Methods \& Software, vol. 30, no. 3, pp. 594-615, 2015.

[31] L. Gómez-Chova, J. Muñoz-Marí, V. Laparra, J. Malo-López, and G. Camps-Valls, "A review of kernel methods in remote sensing data analysis," in Optical Remote Sensing, vol. 3, pp. 171206, Springer, Berlin, Germany, 2011. 


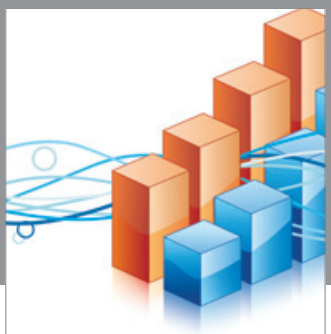

Advances in

Operations Research

vatem alat4

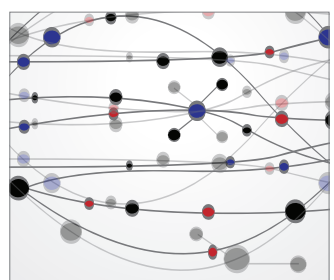

\section{The Scientific} World Journal
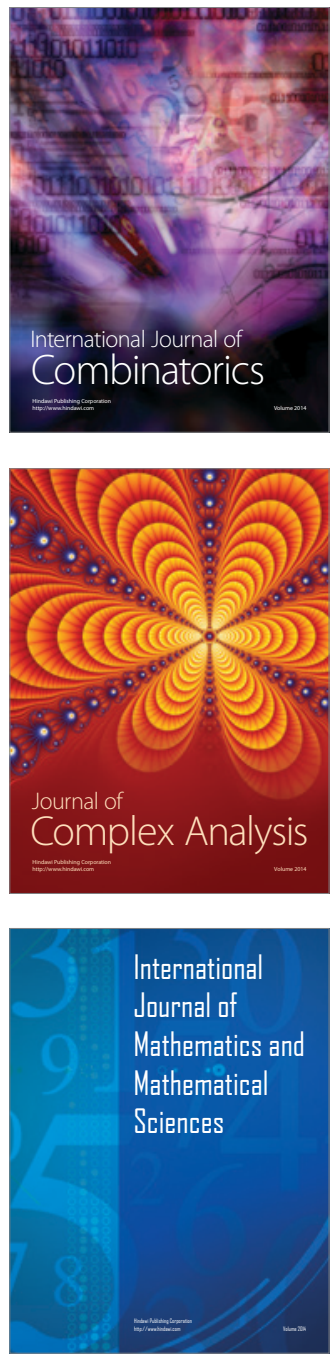
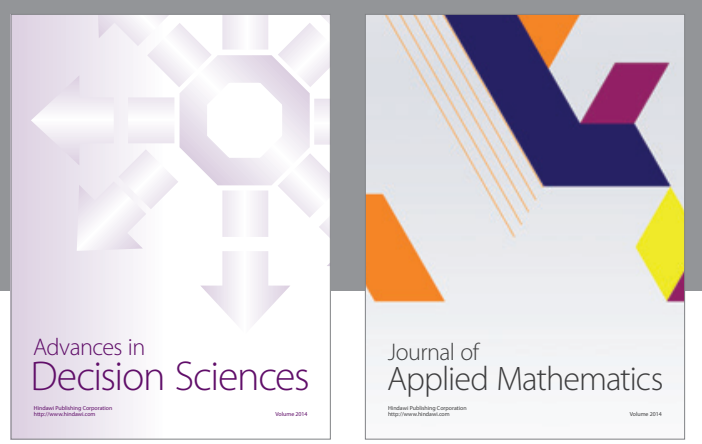

Algebra

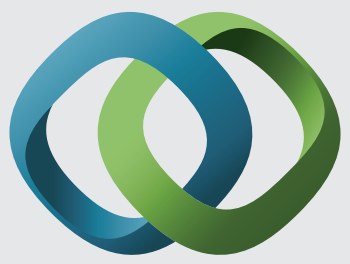

\section{Hindawi}

Submit your manuscripts at

http://www.hindawi.com
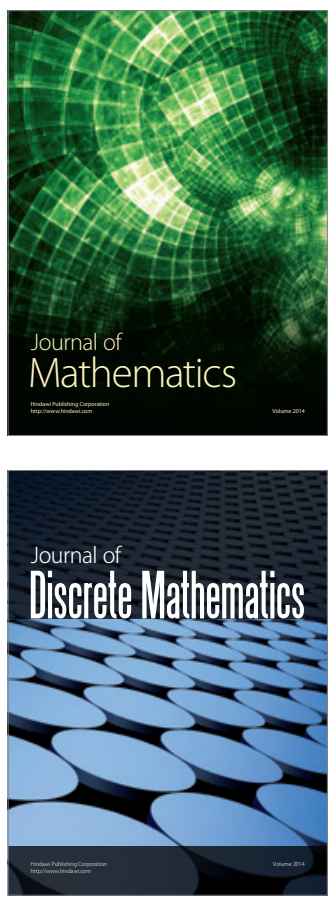

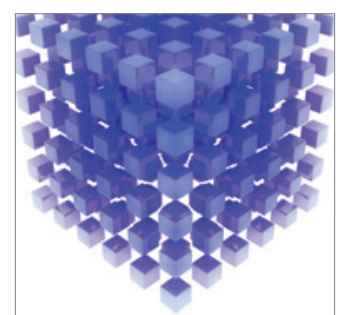

Mathematical Problems in Engineering
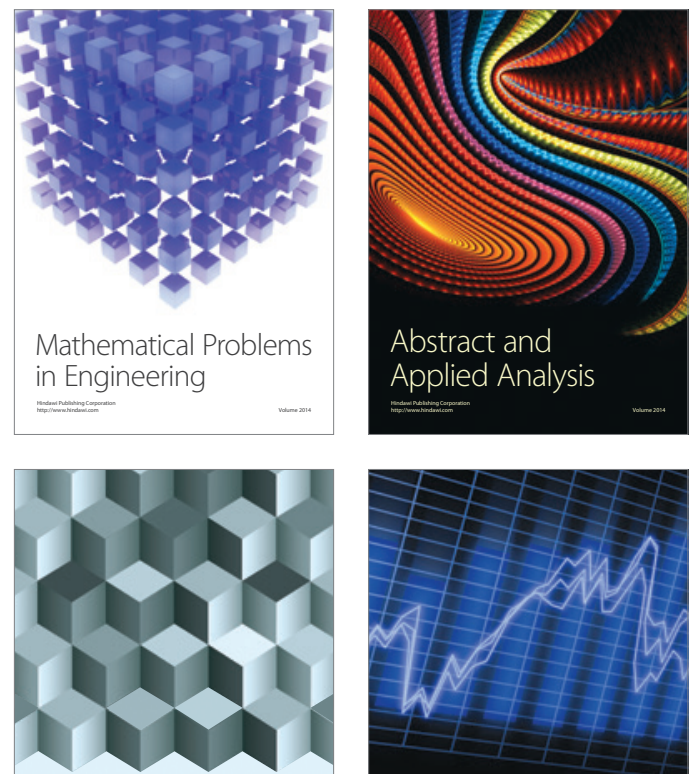

Journal of

Function Spaces

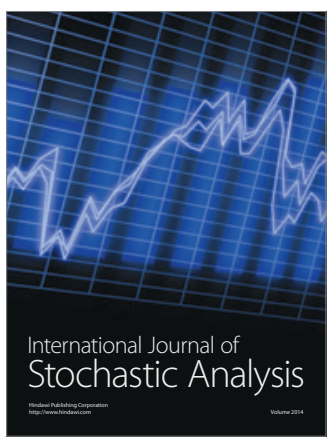

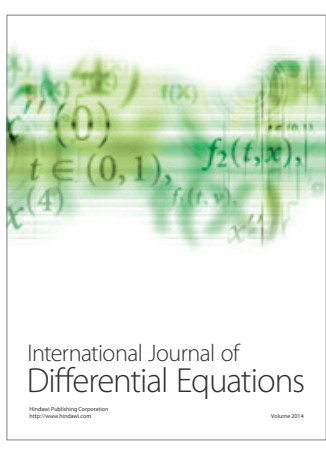
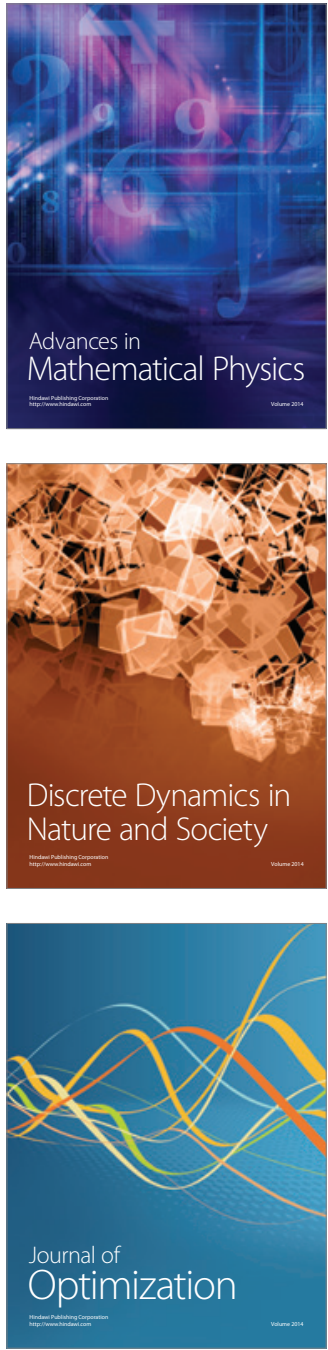